\title{
BMJ Open From breathless to failure: symptom onset and diagnostic meaning in patients with heart failure - a qualitative study
}

\author{
C J Taylor, ${ }^{1}$ F D R Hobbs, ${ }^{1}$ T Marshall, ${ }^{2}$ F Leyva-Leon, ${ }^{3}$ N Gale ${ }^{4}$
}

To cite: Taylor CJ, Hobbs FDR, Marshall T, et al. From breathless to failure: symptom onset and diagnostic meaning in patients with heart failure -a qualitative study. BMJ Open 2017;7:e013648. doi:10.1136/bmjopen-2016013648

- Prepublication history for this paper is available online. To view these files please visit the journal online (http://dx.doi.org/10.1136/ bmjopen-2016-013648).

Received 2 August 2016 Revised 17 December 2016 Accepted 19 January 2017

CrossMark

\footnotetext{
${ }^{1}$ Nuffield Department of Primary Care Health Sciences, University of Oxford, Oxford, UK

${ }^{2}$ Institute of Applied Health Research, University of Birmingham, Birmingham, UK

${ }^{3}$ Aston Medical Research Insitutue, Aston Medical School, Birmingham, UK ${ }^{4}$ Health Services Management Centre, University of Birmingham, Birmingham, UK
}

Correspondence to Dr CJ Taylor; clare.taylor@phc.ox.ac.uk

\section{ABSTRACT}

Objectives: To explore 2 key points in the heart failure diagnostic pathway-symptom onset and diagnostic meaning-from the patient perspective.

Design: Qualitative interview study.

Setting: Participants were recruited from a secondary care clinic in central England following referral from primary care.

Participants: Over age 55 years with a recent ( $<1$ year) diagnosis of heart failure confirmed by a cardiologist following initial presentation to primary care.

Methods: Semistructured interviews were carried out with 16 participants (11 men and 5 women, median age 78.5 years) in their own homes. Data were audiorecorded and transcribed. Participants were asked to describe their diagnostic journey from when they first noticed something wrong up to and including the point of diagnosis. Data were analysed using the framework method.

Results: Participants initially normalised symptoms and only sought medical help when daily activities were affected. Failure to realise that anything was wrong led to a delay in help-seeking. Participants' understanding of the term 'heart failure' was variable and 1 participant did not know he had the condition. The term itself caused great anxiety initially but participants learnt to cope with and accept their diagnosis over time.

Conclusions: Greater public awareness of symptoms and adequate explanation of 'heart failure' as a diagnostic label, or reconsideration of its use, are potential areas of service improvement.

\section{INTRODUCTION}

Heart failure is a clinical syndrome affecting $1 \%$ of adults in developed countries. ${ }^{1}$ It is associated with distressing symptoms, typically breathlessness, fatigue and swollen ankles, and poor outcomes for patients. ${ }^{2-5}$ However, there are drug treatments, device therapies and exercise-based interventions which improve quality of life and survival. ${ }^{6-8}$
Strengths and limitations of this study

- This study is novel in exploring crucial points in the heart failure diagnostic pathway from the patient perspective to identify areas where care may be improved.

- The initial period where participants first experienced symptoms, the point they realised there was something wrong, and the impact this had on help-seeking is described in detail.

- The terminology of 'heart failure', the impact on the patient and the appropriateness of the term itself are questioned.

- The study recruited participants from a single secondary care clinic which may limit generalisability.

Recognising symptoms and making a diagnosis is therefore vital to allow appropriate and timely management.

The diagnosis of most chronic conditions is reliant on patients realising that something is wrong, and seeking help. ${ }^{9}$ The start of many illnesses can, though, be difficult to recognise, and may sometimes only be appreciated in retrospect, once a diagnosis has been reached. ${ }^{10}$ Symptoms vary according to disease and some may be more easily recognised as pathological than others. ${ }^{11}{ }^{12}$ Chronic obstructive pulmonary disease (COPD) is associated with gradual onset of breathlessness which can get worse over time. ${ }^{13}$ Smoking is a causal factor but smokers may initially put their symptoms down to the smoking itself rather than the permanent lung damage which results in COPD. ${ }^{14}$ In contrast, some diseases present with acute symptoms which might be recognised more easily. Acute myocardial infarction may cause crushing central chest pain and patients with stroke may experience a sudden onset of one-sided weakness, speech disturbance or facial asymmetry. Early intervention can lead 
to life-saving treatment and public health campaigns have focused on raising awareness of these symptoms. ${ }^{15} 16$ Unlike myocardial infarction or stroke, heart failure symptoms are often insidious in onset and the patient experience of early symptoms and decision-making around seeking medical attention prior to a diagnosis has not been evaluated.

A diagnostic label is important medically to lead on to an appropriate treatment plan but the terminology used can have an important impact on the patient. They must make sense of their diagnosis and, for chronic conditions, adapt to living with the disease. ${ }^{17}$ According to guidelines, at the end of the diagnostic pathway for heart failure, patients should receive a formal diagnosis which is confirmed by an echocardiogram and explained to them by a specialist. ${ }^{18}$ However, some studies have identified that patients are not necessarily told, or do not retain, the diagnostic label or may not understand what it means. ${ }^{19}$

This study explores the experiences of patients with a recent diagnosis of heart failure with a focus on the symptom onset and diagnosis parts of the pathway to explore how and when patients realised something was wrong and what the term 'heart failure' means to them.

\section{METHODS}

\section{Study design and setting}

Patient experiences of pathways to receiving clinical care are best understood via an in-depth qualitative approach that enables collection of data on attitudes, perceptions and responses. Semistructured interviews were conducted with patients who had recently received a diagnosis of heart failure until saturation of key emergent themes was achieved, that is, additional data were no longer adding new themes to the data set. The interviews were primarily with study participants but the contribution of a relative, if present, was also welcomed.

Participants were recruited from a secondary care heart failure clinic serving a large, socioeconomically diverse population in central England. Patients with a recent $(<1$ year ago) diagnosis of heart failure over the age of 55 who had been referred from primary care were invited for interview. This was to ensure adequate recall of events. Patients not able to give written informed consent or who were too unwell to take part were excluded.

Purposive sampling was planned in order to achieve demographic variation. The aim was to recruit a total of 20 patients over the age of 55 years with at least two participants from each decile age group. A mix of men and women in each age group and patients of white, Asian and black ethnicity were also sought. Arrangements for an interpreter to be used where needed were put in place to prevent exclusion of non-English-speaking patients.

\section{Data collection}

All participants received a patient information sheet and provided written consent. Semistructured interviews, with a duration of 30-60 min, were conducted by the lead author (CJT), a female general practitioner (GP) and clinical researcher trained in qualitative methods. An interview topic guide was used to ensure that key areas were covered to achieve the aims and objectives of the study. Early interviews were reviewed and discussed with NG, an experienced medical sociologist, with expertise in qualitative methods. Minor modifications were made to the interview guide after two interviews, in the light of emerging themes from the data: in particular, further information was sought on the meanings associated with heart failure. All interviews were recorded using a digital voice recorder. The audio files were downloaded to a secure network.

\section{Analysis}

All interviews were transcribed verbatim, then analysed using the framework method. ${ }^{20}{ }^{21}$ Data were anonymised by removing any information which would make the patient identifiable. Transcripts were read and re-read to ensure familiarisation, then initially coded by hand. The coding was reviewed by a second coder (NG), and an independent experienced qualitative research fellow (SS), to ensure that the type and range of codes applied was appropriate. The coding lists were used to develop an analytical framework organised into categories. In total, there were 150 codes organised into 17 categories. All interview transcripts were then coded using the software package NVivo V.10.

Coded data were exported from NVivo according to individual codes and saved as separate documents. The data for each code were read, re-read and then summarised for each of the 16 participants in the study. Microsoft Excel was used to manage the summarised data. A new worksheet was used for each category. Each category was then interpreted using an analytical memo to explore emerging themes and concepts.

\section{RESULTS}

\section{Participants}

Invitation letters were sent out to a total of 100 eligible participants identified by the heart failure clinic. In total, 21 participants returned the reply slip and 16 agreed to take part (1 participant had changed her mind when contacted, 1 had recently been admitted to hospital and 3 participants were not contactable). Between October and December 2014, 15 participants were interviewed in their homes and 1 by telephone. Interviews lasted an average of $42 \mathrm{~min}$ (range 21-74 min). Five women and 11 men participated, their median age was 78.5 years (range 52-87 years) and all but 1 participant was white British. Ten of the 16 interviewees were accompanied by a relative during the interview.

\section{Initial normalisation of symptoms}

Shortness of breath featured in all participant stories. It was often gradual in onset and only noticed to be a 
problem when it interfered with usual daily activities. Breathlessness for many participants was thought to be a normal part of ageing.

I just thought it was probably my age. I don't think I thought it was the heart to start with, no. I just thought that it was because I was walking a long distance and I needed to stop. (P6)

Participants described trying to 'do too much' and thought they were bringing the symptom on themselves by going up a steep incline, going out in bad weather or by 'panicking'. Participants initially coped by resting or 'calming down' to resolve the feeling of breathlessness.

I used to panic and I just couldn't get any breath...When I sat up, it was okay after a short time. I just have to calm myself down because I felt like, am I bringing this on myself by getting in a state? (P5)

Participants had comorbidities and sometimes thought their symptoms were due to their other medical conditions. One participant with COPD thought his breathlessness was because he needed stronger inhalers.

I think perhaps my lungs, COPD. I mean this is what I attach to myself, to thinking, I suppose, for decades now...So I think I must have concentrated and focused on the COPD when it could have been the heart otherwise. (P8)

The degree of breathlessness could be significant before the participant thought it was a problem. One participant had shortness of breath on walking to the toilet a few yards down the hallway and another woke up gasping for breath. Participants carried on with their usual activities despite significant limiting symptoms.

I was going up the hill at the golf course and I can't breathe. I couldn't breathe. It was really bad. I should have stopped, but no. I stood there for a while and got myself up again, hit the ball again. Out of breath again. I should have stopped, but I didn't. I kept on going all the way around, but I was really getting out of breath. (P7)

Some participants had swollen ankles which was a visible persistent reminder, to them and others, that something was wrong.

...swelling of the feet. Which is still going on as you can see there, I end up like a hobbit by the evening. (P4)

Four participants described tiredness, lethargy or a decrease in energy levels which could result in the need for an afternoon nap. For one participant, tiredness was the main symptom but was initially put down to being busy.

I just put it down to the fact that [er] I over stretched myself you know. Yeah I was trying to do too much. Simple simple as that. (P4)
Some participants noticed their symptoms for the first time while away from home-on holiday or staying with relatives-but dismissed their significance putting it down to being outside of their usual environment. A stoical approach prevented participants from taking action to deal with their symptoms.

I thought that's what it was. I was telling myself, "Well I've got to put up with this. It's the medication and I've got to put up with it." So I wasn't doing anything about it. (P7)

\section{Progression of symptoms and realisation that something was wrong}

The timing of initial symptoms ranged from gradual onset over a number of months to a sudden event which required immediate action. Participants took symptoms seriously only once they started to affect activities of daily living.

Anyway we lived on a hill and of course I was starting getting breathless, going up this hill to the shops or whatever, I started getting breathless and I thought, well this is never right, so it went on for a few weeks, and I just went and seen the doctor and he said, "Well I'm going to send you for a test...(P10)

Symptoms often progressed over time. For example, participants noticed their shortness of breath gradually impinging on their activities up to quite severe levels where they were 'gasping for breath' and unable to sleep. One participant called an ambulance after realising he had become so unwell.

I was getting more and more ill...I hadn't realised it because you have a steady decline...You don't feel well one day and not the next...(P15)

One participant just felt ill and did not have a particular explanation for why they felt that way but assumed that the symptoms would probably resolve with time.

I didn't know really, I was mystified, I thought that's unusual and why is this and it probably went on for a couple of months before I went to see the doctor. I thought it would probably pass. (P16)

Participant background influenced the explanation and conclusions they came to. One participant had a nursing background. Being a retired nurse, she thought swollen ankles were due to problems with the heart or kidney-if the swelling could be pressed in with a finger, then it was due to the heart. She did not know exactly what heart problem it was but went to the GP several times as she was worried. Her professional background drove her to feel there was something wrong.

Most participants had retired but many maintained active social lives which were disrupted by the symptoms. One younger participant was working age and initially 
thought that tiredness was a normal part of her busy lifestyle and job, but once her work became affected she sought help.

Most participants were quick to seek medical advice from their GP as soon as they realised something was wrong. Family members often witnessed participants struggling with symptoms and encouraged them to seek help. Until that point, most participants simply did not realise anything was wrong or hoped that the symptoms would pass. A few participants disclosed that, with the benefit of hindsight, they wished they had gone to their doctor sooner and admitted they had known something was wrong but had been reluctant to seek medical advice, instead wanting to carry on as normal.

I should have gone to see him. I should have stopped straight away. Knowing what I know now, what I've been told, I should have stopped and gone straight to see the doctor, but I didn't. (P7)

\section{Variability in understanding of diagnosis}

Participants' understanding of their heart failure diagnosis varied in complexity and depth. Some simply described having a 'weak heart' or 'not getting enough oxygen in the blood' while others gave more detailed descriptions. One participant had seen a cardiologist, attended the nurse-led heart failure clinic and had responded to take part in the heart failure study but, even on direct questioning during the interview, did not realise that he had heart failure. Participant education and background appeared to influence understanding and sense-making of the diagnosis. Two participants were engineers by background and had a detailed understanding of the disease process by thinking of heart failure in mechanical terms.

...it's a pump system isn't it that's all it is, it's a pump system. You've got the power of the pump, you've got the pipework going round...if the pipework gets constricted in any way then the pressure will go up. There [is] an easy hydraulic relationship with that...(P4)

Some participants were confused by the diagnosis but did not necessarily want any further clarification.

They tried, but they used so much jargon, I didn't understand. I didn't want to know too much, anyway. But I saw what was written down and was able to translate that... They say my left ventricle is not working fully. They don't say why...The last thing I do is worry. There's a hospital full of doctors. Let them do it. (P9)

Some participants actively sought extra knowledge about the diagnosis by looking online or in textbooks while others preferred to avoid knowing too much.

I didn't bother to go too deeply into it, you know knowledge can kill you, the wrong kind of knowledge, or half knowledge...(P8)
One participant was still working and found she needed an explanation for her condition which she could tell work colleagues to account for why she gets so tired.

I just tell them it's a heredity thing, I've got a bit of heart failure and I get more tired than what you do. As I said it's probably being twice as tired and working twice as hard as what they do. (P13)

\section{Fear and uncertainty caused by heart failure terminology}

There was a degree of uncertainty caused by the use of some terminology. The term 'heart failure' itself was often associated with fear and a concern that the heart may stop or that outlook was poor.

Well I mean heart failure means, when they [say] heart failure it means your heart's going to, it's wearing out or whatever, it's going to stop. It could stop. It's failing. And I know you've only got one so I mean let's face it that's not a good thing. (P2)

There was also some confusion over ejection fraction terminology and what that meant-if ejection fraction was $20 \%$, for example, one participant assumed that meant only $20 \%$ of their heart was working and was not aware that a normal ejection fraction is $50 \%$ or above. Despite the fear and lack of clarity, participants learnt to cope with their anxieties over time.

It was worrying I mean you know when they tell you that your heart's not working properly and they call it 'heart failure', whatever's wrong with your heart they call it heart failure don't they which I think is horrible because failure means it could stop you know. That's worrying but the longer you live with it, the more you get used to it. (P2)

Some participants recalled that the term had not been used initially and was introduced later on by the specialist team delivering the diagnosis.

It was very good, I mean he says, it's just a degree of heart failure. They actually didn't use those words for a long time to me, he just said...he told me it was a left valve that wasn't pumping properly. It should pump at so much percentage and it was pumping at a lesser percentage and that's why you're feeling tired...He explained to me quite well...(P13)

\section{Relationship between initial symptoms and heart failure diagnosis}

Before being given a diagnosis, none of the participants, even those with a history of cardiovascular disease, suspected that their initial symptoms were linked to previous cardiac events. However, when they were told they had heart failure, some participants suggested their own reasons for why they had developed the condition while others recalled being given a particular explanation by the specialists. 
But a part of my heart is damaged, he says, which is caused by a heart attack I suppose. (P10)

One participant blamed himself for 'over doing it' and was convinced his heart failure had been brought on by his own behaviour.

I think this is one of the reasons I've, over the years, probably just wore myself out...I'm just like an engine, just blowing up, you know. (P7)

Several participants reported improvement or resolution of their symptoms following diagnosis. For these participants, a confirmed diagnosis had led to appropriate treatment which relieved unpleasant symptoms they had experienced for some time.

They say to me, "Do you sit up in bed at night? Or do you wake up gasping?” I don't. I'm really well, really well; I think they've done a marvellous job on me. (P11)

\section{DISCUSSION}

\section{Summary}

Participants in the study did not realise initially that the symptoms of breathlessness, ankle swelling or tiredness were due to heart failure. Instead, they thought their symptoms were due to normal ageing, 'doing too much', other medical conditions and medications or just assumed that they would pass. Symptoms were gradual in onset and often severe, but it was not until they had an impact on activities of daily living that participants sought medical help. For some, a change in circumstance, such as going on holiday, meant they noticed their limitations for the first time. Participants' understanding of 'heart failure' as a term varied and was associated with fear and uncertainty. Some participants could describe the mechanisms of heart failure in terms of a failing pump system, and relate this to previous heart damage, whereas other participants chose not to consider the diagnosis in considerable detail. One participant in the study did not even know that he had heart failure.

\section{Strengths and limitations}

This study is unique in exploring patient experiences of heart failure from initial symptoms to receiving a formal diagnosis. Participants were recruited from a cardiology outpatient clinic where their diagnosis was confirmed after referral by their GP. Recruiting participants from a single outpatient clinic may have introduced a selection bias and could limit the generalisability to other heart failure services; however, the findings are unlikely to be isolated to patients attending this clinic, particularly as many of the observations have been linked to established theoretical concepts (such as normalisation of symptoms and biographical disruption-discussed below) that have been described in other chronic conditions. ${ }^{22} 23$

The original intention was to use purposive sampling to ensure that participants were from a range of ages, gender and ethnic groups. Challenges in recruitment, including a poor response rate to the study invitation letter, limited the number of participants available for the study. There were participants from each decile from 50 to 90 years and a mix of men and women; however, only one of the 16 participants was from a minority ethnic group. All participants also spoke English as their primary language. The experiences of patients from different ethnic groups and non-English speakers were not captured in this study. The findings are therefore less likely to be relevant to this group.

The professional role of the interviewer can influence interactions during the interview. ${ }^{24}$ The researcher conducting the interviews in this study was a GP which could have influenced the behaviour of participants. ${ }^{25}$ Various steps were taken in the study design, data collection and analysis to enable reflexive research practice, such as undertaking pilot interviews that were discussed with the wider team, and cross-checking of coding and data analysis with an experienced qualitative researcher. In addition, several steps were taken during the research process to reduce the observer effect including use of the interviewer's first name with participants, and emphasising that the interviewer was there as a researcher rather than in a GP role. These steps have enhanced the credibility of the findings. ${ }^{26}$

\section{Comparison with the existing literature \\ Normalisation}

Normalisation of symptoms is a common sociological phenomenon and a barrier to diagnosis in other medical conditions, such as dementia where 'forgetfulness' is initially seen as a normal part of ageing. ${ }^{27}$ The presence of symptoms in the general population is common which might explain why 'normalising' prevalent symptoms such as fatigue or breathlessness is so widespread. A study by Petrie $e t a l^{28}$ examined the prevalence of symptoms in a randomly selected sample of 1000 participants from the general population. Researchers asked participants if they had experienced any of a list of 46 symptoms in the previous 7 days. Thirteen per cent had experienced shortness of breath and $36 \%$ had experienced fatigue. The challenge therefore is to enable patients to identify symptoms which are significant and associated with a pathological disease process rather than within normal limits.

Many participants in this heart failure study had a history of previous cardiovascular disease and had multiple past contacts with healthcare. Epidemiological evidence shows that patients who have had a previous myocardial infarction are more likely to develop heart failure than those who have not had a previous event. ${ }^{2}$ Yet participants were initially unaware that their symptoms could be due to heart failure.

\section{Help-seeking and biographical disruption}

The concept of 'biographical disruption' was first described by Bury in the 1980s. He interviewed patients 
with a new diagnosis of rheumatoid arthritis and found that the symptoms, and ultimately the label of disease, had a profound effect on the patient's social world and their sense of self. ${ }^{25}$ In the interviews with patients with heart failure, participants reflected that their whole world changed at the point where they realised something was wrong and sought medical help.

A narrative review by Gravely-Witte $e t a l^{29}$ explored the length of delay in seeking medical care for patients with heart failure-admitted to hospital rather than presenting to primary care-according to symptom type and onset. Participants who suddenly developed shortness of breath were less likely to delay seeking help than those with symptoms of more gradual onset such as ankle swelling or fatigue. The community population with new onset of chronic heart failure is likely to differ from the population with existing heart failure experiencing an exacerbation which requires hospital admission; however, the impact of symptom type on delay in seeking medical help within primary care is worthy of further consideration in future research.

Symptom worsening, effect on activities of daily living and family concern were all reasons for participants in the study to see their GP. A qualitative synthesis by Smith et $a l,{ }^{30}$ exploring patients' help-seeking experiences and delay in cancer presentation, found remarkable similarities in their list of triggers to recognition of illness which included 'symptoms worsen or do not go away', 'symptoms affect everyday life' and 'discussion of symptoms with friends and family'. Other triggers which were not found in the current study were 'specific well-known symptoms (eg, lump)' and 'knowledge of cancer symptoms and awareness of risk'. These concepts were notably absent from all of the interviews in the heart failure study suggesting that awareness of heart failure symptoms is much less than for cancer symptoms in the general population. National public health campaigns in England have focused on cancer symptoms in recent years which may explain some of the disparity. ${ }^{31}$

\section{Understanding the diagnosis}

In this study, participants were told that they had a diagnosis of heart failure within secondary care. Across Europe, the place of diagnosis varies between countries. Hobbs et $a l^{32}$ interviewed 1363 primary care physicians from 14 European countries and reviewed 11062 patient notes and found that overall $50 \%$ of heart failure diagnoses were made in primary care. Delivering the news of a life-changing diagnosis is a key communication skill for clinicians. ${ }^{33}$ For some, a timely diagnosis leading to treatment and rapid symptom relief can be welcome while for others the news can be devastating. Discussions need to be tailored to the individual and based on preexisting knowledge and understanding as well as their wishes for what they want to be told about their diagnosis. The current evidence base focuses on interventions for practitioners to enhance their communication skills but more research is required to establish how delivery of diagnosis impacts on patient-reported outcomes, and how this can be improved. ${ }^{34}$

Bury's concept of biographical disruption was originally developed in the context of a new diagnosis of rheumatoid arthritis, but the concept has been found to apply to many chronic illnesses including COPD and cancer. $^{35}$ Heart failure has a poorer prognosis than many cancers. A recent study exploring the 'disruption' of a cancer diagnosis with a poor outlook found that participants acknowledged that a full recovery was impossible, with their self-identity and social world already changed forever, and instead aimed to achieve a 'new normal' within the limits of what their disease allowed. ${ }^{36}$ This study adds to the literature a new context in which the concept of biographical disruption has salience.

Rodriguez et $a l^{37}$ interviewed American patients about their knowledge and understanding of a heart failure diagnosis. Patients reported a need for more information about heart failure and a greater opportunity to discuss their condition in detail with their physician. Patients also expressed a desire to discuss prognosis but reported that this was lacking during medical consultations.

Most participants in this UK-based study had not been given any written information, so some went online to find out more about their condition. However, participants would have preferred information tailored to their own particular condition and circumstance and would have welcomed more time to discuss the diagnosis with the secondary care team, perhaps at a further appointment. In a review by Davidson ${ }^{38}$ examining tools for conversations about treatment decisions and outlook in patients with heart failure, the 'receptivity' of the patient to information about their conditions was not uniform. Patients valued honesty in general but the depth and type of information they required was highly variable. An individualised approach to delivering the diagnosis and providing further information is therefore required.

\section{Implications for practice and research}

Patients developed plausible and complex explanations for their symptoms which delayed their presentation to primary care. Increased awareness of heart failure symptoms is vital for patients, carers and the public to recognise the condition. Campaigns by government and charities have raised awareness of the symptoms of several different cancers with the hope of earlier diagnosis leading to better outcomes. A similar campaign at a national level, or a more targeted campaign aimed at those with a previous history of myocardial infarction, may help encourage patients with possible heart failure to access healthcare earlier. This would have a large associated cost, so further research in this area to consider effectiveness is warranted.

The nomenclature used around heart failure is complex and does not perhaps accurately describe the pathological processes involved in deterioration of 
cardiac function. ${ }^{39}$ In recent years, the emergence of a new type of heart failure where the ejection fraction is within normal limits-heart failure with preserved ejection-has led to debate about the classification which should be used by specialists for consistency. ${ }^{40}$ In guidelines, there has been an emphasis on publishing precise definitions required for heart failure diagnosis and there has also been a suggestion by some authors to 'stage' the disease in a similar way to cancer. ${ }^{41}$ However, for patients, the term 'heart failure' itself is associated with fear and uncertainty. Understanding the diagnosis, and any underlying cause, may be important for future engagement in treatment decisions, adherence and selfmanagement. Currently, guidelines recommend a patient-centred approach including an assessment of their prior knowledge, what the patient would like to know and involvement of carers. There is little research on the impact of the terminology used in heart failure and a change in the name to a less frightening and better descriptive term may be helpful for patients and clinicians.

\section{CONCLUSION}

This study revealed that patients with heart failure initially normalised their symptoms which delayed their presentation to primary care. Increased public awareness of heart failure symptoms may help patients to recognise the disease earlier. Other methods such as screening might effectively identify patients earlier in the disease process. The term 'heart failure' is unhelpful and further work is required to establish what terminology patients might find acceptable and the feasibility of changing the terms routinely used in healthcare. Any change in terminology would require a global consensus.

\section{Twitter Follow Clare Taylor @clarejtaylor}

Acknowledgements The authors would like to acknowledge the heart failure clinic team for their role in patient recruitment, Sonal Shah (an experienced qualitative research fellow) for independently cross-checking the coding framework and all of the participants who shared their experiences in such an open and generous way.

Contributors CJT conceived the idea, wrote the study protocol, obtained ethical approval, carried out interviews, analysed the data and drafted the manuscript. NG provided expert supervision and contributed to the protocol, analysis and drafting of the manuscript. FL-L was the local principal investigator for the study. FDRH and TM provided critical comment throughout. All authors have approved the final manuscript.

Funding This study was funded through a Doctoral Research Fellowship (DRF-2012-05-407) awarded to CJT from the National Institute for Health Research (NIHR). TM is partly funded by the NIHR through the Collaborations for Leadership in Applied Health Research and Care for West Midlands (CLAHRC-WM). FDRH is partly funded, through supervision sessions, by the NIHR School for Primary Care Research, NIHR Oxford Biomedical Research Centre, NIHR Collaboration for Leadership in Applied Health Research and Care Oxford, and Harris Manchester College, University of Oxford.

Disclaimer The views expressed are those of the authors and not necessarily those of the NHS, the NIHR, the Department of Health, NHS Partner Trusts, University of Birmingham or the CLAHRC-BBC Management Group.
Competing interests None declared.

Ethical approval This study received ethical approval from the Hampshire A Research Ethics Committee (reference 13/SC/0475).

Provenance and peer review Not commissioned; externally peer reviewed.

Data sharing statement The original data are available from the corresponding author.

Open Access This is an Open Access article distributed in accordance with the Creative Commons Attribution Non Commercial (CC BY-NC 4.0) license, which permits others to distribute, remix, adapt, build upon this work noncommercially, and license their derivative works on different terms, provided the original work is properly cited and the use is non-commercial. See: http:// creativecommons.org/licenses/by-nc/4.0/

\section{REFERENCES}

1. Mosterd A, Hoes AW. Clinical epidemiology of heart failure. Heart 2007;93:1137-46.

2. Roger VL. Epidemiology of heart failure. Circ Res 2013;113:646-59.

3. Barker WH, Mullooly JP, Getchell W. Changing incidence and survival for heart failure in a well-defined older population, 1970-1974 and 1990-1994. Circulation 2006;113:799-805.

4. Bleumink GS, Knetsch AM, Sturkenboom MCJM, et al. Quantifying the heart failure epidemic: prevalence, incidence rate, lifetime risk and prognosis of heart failure: the Rotterdam Study. Eur Heart $J$ 2004;25:1614-19.

5. Stewart S, Jenkins A, Buchan S, et al. The current cost of heart failure to the National Health Service in the UK. Eur J Heart Fail 2002;4:361-71.

6. Dickstein K. Diagnosis and assessment of the heart failure patient: the cornerstone of effective management. Eur $J$ Heart Fail 2005; 7:303-8.

7. Rutten $\mathrm{FH}$, Cramer MJ, Grobbee DE, et al. Unrecognized heart failure in elderly patients with stable chronic obstructive pulmonary disease. Eur Heart J 2005;26:1887-94.

8. Le Jemtel TH, Padeletti M, Jelic S. Diagnostic and therapeutic challenges in patients with co-existent chronic obstructive pulmonary disease and chronic heart failure. J Am Coll Cardiol 2007;49: 171-80.

9. White $\mathrm{C}$, Lentin $\mathrm{P}$, Farnworth L. Multimorbidity and the process of living with ongoing illness. Chronic IIIn 2016;12:83-97.

10. Molbaek K, Horslev-Petersen K, Primdahl J. Diagnostic delay in rheumatoid arthritis: a qualitative study of symptom interpretation before the first visit to the doctor. Musculoskelet Care 2016;14:26-36.

11. Erritty $\mathrm{P}$, Wydell TN. Are lay people good at recognising the symptoms of schizophrenia? PLOS ONE 2013;8:e52913.

12. Dumit J. Illnesses you have to fight to get: facts as forces in uncertain, emergent illnesses. Soc Sci Med 2006;62:577-90.

13. Brenner S, Guder G. The patient with dyspnea. Rational diagnostic evaluation. Herz 2014;39:8-14.

14. Hayward RA, Chen $\mathrm{Y}$, Croft $\mathrm{P}$, et al. Presentation of respiratory symptoms prior to diagnosis in general practice: a case-control study examining free text and morbidity codes. BMJ Open 2015;5: e007355.

15. Henriksson C, Lindahl B, Larsson M. Patients' and relatives' thoughts and actions during and after symptom presentation for an acute myocardial infarction. Eur J Cardiovasc Nurs 2007;6:280-6.

16. Wolters FJ, Paul NL, Li L, et al., Oxford Vascular Study. Sustained impact of UK FAST-test public education on response to stroke: a population-based time-series study. Int J Stroke 2015;10:1108-14.

17. Heaton J, Räisänen U, Salinas M. 'Rule your condition, don't let it rule you': young adults' sense of mastery in their accounts of growing up with a chronic illness. Sociol Health IIIn 2016;38:3-20.

18. European Society of Cardiology. European Society of Cardiology guidelines for the diagnosis and treatment of acute and chronic heart failure 2016. Eur Heart J 2016;37:2129-200.

19. Andersson L, Eriksson I, Nordgren L. Living with heart failure without realising: a qualitative patient study. $\mathrm{Br} J$ Community Nurs 2012;17:630-7.

20. Ritchie J, Lewis J. Qualitative research practice: a guide for social science students and researchers. London: Sage Publications, 2003.

21. Gale NK, Heath G, Cameron E, et al. Using the framework method for the analysis of qualitative data in multi-disciplinary health research. BMC Med Res Methodol 2013;13:117. 
22. McCabe PJ, Rhudy LM, DeVon HA. Patients' experiences from symptom onset to initial treatment for atrial fibrillation. J Clin Nurs 2015;24:786-96.

23. Bury M. Chronic illness as biographical disruption. Sociol Health IIIn 1982;4:167-82.

24. Richards H, Emslie C. The 'doctor' or the 'girl from the University'? Considering the influence of professional roles on qualitative interviewing. Fam Pract 2000;17:71-5

25. McNair R, Taft A, Hegarty K. Using reflexivity to enhance in-depth interviewing skills for the clinician researcher. BMC Med Res Methodol 2008;8:73.

26. Mays N, Pope C. Qualitative research in health care. Assessing quality in qualitative research. BMJ 2000;320:50-2.

27. Bunn F, Goodman C, Sworn K, et al. Psychosocial factors that shape patient and carer experiences of dementia diagnosis and treatment: a systematic review of qualitative studies. PLoS Med 2012;9:e1001331.

28. Petrie KJ, Faasse K, Crichton $\mathrm{F}$, et al. How common are symptoms? Evidence from a New Zealand national telephone survey. BMJ Open 2014;4:e005374

29. Gravely-Witte $\mathrm{S}$, Jurgens $\mathrm{CY}$, Tamim $\mathrm{H}$ et al. Length of delay in seeking medical care by patients with heart failure symptoms and the role of symptom-related factors: a narrative review. Eur J Heart Fail 2010;12:1122-9.

30. Smith LK, Pope C, Botha JL. Patients' help-seeking experiences and delay in cancer presentation: a qualitative synthesis. Lancet 2005;366:825-31.

31. Public Health England. Be Clear on Cancer campaign. http://www.nhs. uk/NHSEngland/NSF/Pages/NAEDI.aspx (accessed 18 Jul 2016).
32. Hobbs FD, Korewicki J, Cleland JG, et al. The diagnosis of heart failure in European primary care: the IMPROVEMENT programme survey of perception and practice. Eur $J$ Heart Fail 2005;7:768-79.

33. General Medical Council. Good Medical Practice. http://www. gmc-uk.org/guidance/good_medical_practice.asp (accessed 18 Jul 2016).

34. Paul CL, Clinton-McHarg T, Sanson-Fisher RW, et al. Are we there yet? The state of the evidence base for guidelines on breaking bad news to cancer patients. Eur J Cancer 2009;45:2960-6.

35. Jowsey T, Yen LE, Bagheri N, et al. Time spent by people managing chronic obstructive pulmonary disease indicates biographical disruption. Int J Chron Obstruct Pulmon Dis 2014;9:87-97.

36. Balmer C, Griffiths F, Dunn J. A 'new normal': exploring the disruption of a poor prognostic cancer diagnosis using interviews and participant-produced photographs. Health (London) 2015;19:451-72.

37. Rodriguez KL, Appelt CJ, Switzer GE, et al. "They diagnosed bad heart': a qualitative exploration of patients' knowledge about and experiences with heart failure. Heart Lung 2008;37:257-65.

38. Davidson PM. Difficult conversations and chronic heart failure: do you talk the talk or walk the walk? Curr Opin Support Palliat Care 2007;1:274-8.

39. Redfield MM, Kitzman DW. Heart failure: a rose by any other name? Congest Heart Fail 2006;12:166-8

40. Sanderson JE. HFNEF, HFpEF, HF-PEF, or DHF: what is in an acronym? JACC Heart Fail 2014;2:93-4.

41. Fedele F, Severino P, Calcagno S, et al. Heart failure: TNM-like classification. J Am Coll Cardiol 2014;63:1959-60. 